\title{
ELAVL1 is transcriptionally activated by FOXC1 and promotes ferroptosis in myocardial ischemia/reperfusion injury by regulating autophagy
}

\author{
Hui-Yong Chen ${ }^{1,2}$, Ze-Zhou Xiao ${ }^{1}$, Xiao Ling ${ }^{1}$, Rong-Ning Xu' ${ }^{1}$, Peng Zhu ${ }^{1 *}$ and Shao-Yi Zheng ${ }^{1 *}$ (D)
}

\begin{abstract}
Aims: Myocardial ischemia is the most common form of cardiovascular disease and the leading cause of morbidity and mortality. Understanding the mechanisms is very crucial for the development of effective therapy. Therefore, this study aimed to investigate the functional roles and mechanisms by which ELAVL1 regulates myocardial ischemia and reperfusion (I/R) injury.

Methods: Mouse myocardial I/R model and cultured myocardial cells exposed to hypoxia/reperfusion (H/R) were used in this study. Features of ferroptosis were evidenced by LDH activity, GPx4 activity, cellular iron, ROS, LPO, and GSH levels. The expression levels of autophagy markers (Beclin-1, p62, LC3), ELAVL1 and FOXC1 were measured by qRT-PCR, immunostaining and western blot. RIP assay, biotin-pull down, ChIP and dual luciferase activity assay were employed to examine the interactions of ELAVL1/Beclin-1 mRNA and FOXC1/ELAVL1 promoter. CCK-8 assay was used to examine viability of cells. TTC staining was performed to assess the myocardial I/R injury.

Results: Myocardial I/R surgery induced ferroptosis and up-regulated ELAVL1 level. Knockdown of ELAVL1 decreased ferroptosis and ameliorated I/R injury. Si-ELAVL1 repressed autophagy and inhibition of autophagy by inhibitor suppressed ferroptosis and I/R injury in myocardial cells. Increase of autophagy could reverse the effects of ELAVL1 knockdown on ferroptosis and I/R injury. ELAVL1 directly bound with and stabilized Beclin-1 mRNA. Furthermore, FOXC1 bound to ELAVL1 promoter region and activated its transcription upon $\mathrm{H} / \mathrm{R}$ exposure.

Conclusion: FOXC1 transcriptionally activated ELAVL1 may promote ferroptosis during myocardial I/R by modulating autophagy, leading to myocardial injury. Inhibition of ELAVL1-mediated autophagic ferroptosis would be a new viewpoint in the treatment of myocardial I/R injury.
\end{abstract}

Keywords: Myocardial ischemia reperfusion, Ferroptosis, Autophagy, ELAVL1, FOXC1

\section{Introduction}

Myocardial ischemia occurs when the blood flow to the heart is clogged or reduced, resulting in impaired

\footnotetext{
*Correspondence: zhupengg664@163.com; zsheoyii461@163.com 1 Department of Cardiovascular Surgery, Nanfang Hospital, Southern Medical University, No.1838 North Guangzhou Avenue, Baiyun District, Guangzhou 510515, Guangdong, People's Republic of China

Full list of author information is available at the end of the article
}

oxygen and nutrient supply to heart tissues like cardiac muscle and subsequent heart dysfunction (Frank 2012). It is the most common form of coronary heart disease, and the leading cause to morbidity and mortality worldwide (Aguero 2015). Proper and timely restoration of the blood flow through anti-thrombolytic drugs or mechanical interventions is the primary treatment ( $\mathrm{Lu}$ et al. 2015). However, those treatments are restricted by a 
narrow time window and side effects. Moreover, the reperfusion process would induce a secondary injury. Many mechanisms have been proposed to mediate the reperfusion-induced injury, including inflammation and altered metabolism (Frank 2012; Hausenloy and Yellon 2013; Turer and Hill 2010). Nevertheless, due to its complexity, a clear picture is still lacking. Given the current situation, it is very necessary to understand the molecular mechanism of myocardial ischemia/reperfusion (I/R) injury so that effective therapeutic strategies could be developed.

Ferroptosis is a novel form of programmed cell death featured by a great amount of cellular iron and lipid peroxides ( $\mathrm{Li}$ 2020). The process is iron-dependent and involves an enhanced accumulation of lipid reactive oxygen species (ROS) and a compromised glutathione (GSH)-dependent antioxidant system (Xie 2016). Further, previous studies have shown that ferroptosis is connected to autophagy, a conserved process that helps maintain cell homeostasis by degrading dysfunctional organelles and proteins in the autophagosomes, and to some extent ferroptosis is dependent on autophagy (Zhou 2019a, 2019b). Autophagy is largely involved in and significantly contributes to the myocardial I/R injury (Wu et al. 2019). In addition, emerging evidence implicates a critical role of ferroptosis in myocardial I/R injury as iron deposition and overproduction of ROS have been observed during diabetic myocardial damage (Li et al. 2020), although the exact role of ferroptosis in myocardial $I / R$ injury is not well understood.

ELAVL1 (embryonic lethal-abnormal vision like protein 1) is an AU-rich element (ARE) and U-rich element RNA binding protein (RBP) that regulates gene expression by stabilizing message RNAs (mRNAs) (Simone and Keene 2013). By binding with the $3^{\prime}$ UTR of target mRNAs, ELAVL1 promotes the expression of many genes, such as VEGF-A, an angiogenic factor, and TNF $\alpha$ (Levy et al. 1998; Wang 2013). As a result, ELAVL1 plays a crucial role in many cellular processes, including angiogenesis, apoptosis, and inflammation (Wang 2013; Srikantan and Gorospe 2012). Previous studies have reported an elevation in ELAVL1 level in human diabetic heart and implicated its role in cardiac pyroptosis, as well as ferroptosis in liver fibrosis (Bruin et al. 2017; Zhang 2018). Further, knockdown of ELAVL1 attenuates inflammatory responses during myocardial infarction (Krishnamurthy 2010). We, therefore, hypothesized that ELAVL1 might contribute to myocardial $I / R$ injury, despite that the relevant studies of the role of ELAVL1 in myocardia $\mathrm{I} / \mathrm{R}$ are limited.

Forkhead box $\mathrm{C} 1$ (FOXC1) is a transcription factor that belongs to the FOX family featured by the conserved "fork-head" or "winged-helix" DNA-binding domain (Hannenhalli and Kaestner 2009). It has diverse functions like cell growth, metabolism, and survival (Hannenhalli and Kaestner 2009). Some previous studies indicate that FOXC1 is a hypoxia-activated transcription factor to promote cancer cell growth (Lin 2017). In addition, some literature suggests that FOXC1 could be induced during myocardial ischemia (Zhang 2019). The underlying mechanisms of FOXC1 activation during myocardial I/R are elusive. Our preliminary bioinformatic analysis suggested that FOXC1 may bind ELAVL1 promoter. We thus hypothesized that ELAVL1 might be a downstream effector of FOXC1 in myocardial I/R.

In the present study, we sought to investigate the function of ferroptosis in myocardial I/R injury by focusing on FOXC1/ELAVL1 interaction. We confirmed that FOXC1 transcriptionally activated ELAVL1 greatly contributes to myocardial I/R injury via increasing autophagic ferroptosis. Our study provides insights into the mechanisms of myocardial $\mathrm{I} / \mathrm{R}$ and avenues for the development of future therapy.

\section{Materials and methods \\ Mouse myocardial ischemia/reperfusion (I/R) model}

All animal experiments have been approved by the Committee on Animal Care and Use of Nanfang Hospital, Southern Medical University and were performed according to the guidance. Healthy male C57BL/6 J mice (8 weeks) were purchased from Shanghai SLAC laboratory Animal Co., Ltd. (Shanghai, China) and kept in the standard animal facility. To induce myocardial I/R injury, mice were anesthetized by ketamine and xylazine ( 250 and $10 \mathrm{mg} / \mathrm{kg}$, respectively) first and maintained on $3 \%$ isoflurane. An abdominal incision was made around the fourth intercostal space and the left anterior descending coronary artery (LAD) was exposed. LAD was ligated with the silk suture for $1 \mathrm{~h}$ followed by $4 \mathrm{~h}$ of perfusion. During the perfusion, the incision was closed. For the sham group, same surgery procedures were performed without any ligation. For inhibition of ELAVL1 or overexpression of Beclin- 1 in mice, lentivirus vectors $\left(1 \times 10^{8}\right.$ titers) obtained from GeneChem (Shanghai, China) were used for left ventricular cavity injection. After 7 days of lentivirus infection, mice were subjected to myocardial I/R surgery.

\section{Cell culture and treatments}

Human cardiomyocytes (HCM cells) were obtained from American Type Culture Collection (ATCC, Manassas, VA, USA) and used for the experiments. Cells were cultured in Dulbecco's modified Eagle's medium (DMEM; Sigma-Aldrich, USA) supplemented with $10 \%(\mathrm{v} / \mathrm{v})$ fetal bovine serum (FBS; GIBCO, USA) and $2 \mathrm{mM}$ glutamine (GIBCO), and maintained at $37^{\circ} \mathrm{C}$ in a humidified incubator containing $5 \% \mathrm{CO}_{2}$. For overexpression 
of Beclin-1, Beclin-1 cDNA was cloned into the overexpression construct (pcDNA3.1). Small interfering RNA of ELAVL1 (si-ELAVL1) and FOXC1 (si-FOXC1), negative control (NC) were synthesized and purchased from GeneChem. The transfection was performed using Lipofectamine 3000 (Invitrogen, Missouri, USA) according to the manufacturer's guidance. Briefly, cells were grown up to $60-80 \%$ confluence and then added with $1 \mu \mathrm{g}$ plasmid together with $1 \mu \mathrm{L}$ Lipofectamin 3000 . To mimic the myocardial I/R injury in vitro, HCM cells were subjected to hypoxia followed by reoxygenation (H/R). Briefly, cells were placed in an anaerobic chamber $\left(95 \% \mathrm{~N}_{2}\right.$ and $5 \%$ $\mathrm{CO}_{2}$ ) at $37{ }^{\circ} \mathrm{C}$ for $2 \mathrm{~h}$. Subsequently, the cells were put back in the normal culture condition with $95 \%$ air and $5 \%$ $\mathrm{CO}_{2}$ for another $24 \mathrm{~h}$. Control cells were cultured under normal culture conditions.

\section{Cell counting kit-8 (CCK-8) assay}

Cell proliferation was measured using the standard CCK-8 kit according to the manufacturer's instruction. Cells were plated in the 96-well plates and cultured in the incubator. $10 \mu \mathrm{L}$ CCK-8 solution was added to each well and incubated at $37^{\circ} \mathrm{C}$ for $2 \mathrm{~h}$. The absorbance at $450 \mathrm{~nm}$ was analyzed with the standard microplate reader.

\section{Iron level detection assay}

Intracellular iron level was determined with the standard Iron assay kit (Abcam, USA) according to the manufacturer's protocol. Cultured or transfected cells following treatments were lysed with RIPA lysis buffer supplemented with protease inhibitor. Protein concentration in extracted lysate was quantified with the pierce BCA protein Assay (Thermo Fisher Scientific, USA). Iron levels in the extracted lysate was similarly quantified with the Iron assay kit. Iron level in protein lysate was calculated. The iron level detection of tissues was the same as above.

\section{Cellular ROS detection assay}

ROS level was determined with the standard ROS detection kit (ab113851; Abcam, USA) based on the manufacturer's protocol. The HCM cells were plated into 96-well culture plates at a density of $2.5 \times 10^{4}$ cells/well. Cultured or transfected cells following treatments were incubated with the ROS working solution at $37{ }^{\circ} \mathrm{C}$ for $45 \mathrm{~min}$ in the dark and the fluorescence intensity was analyzed.

\section{Cellular glutathione (GSH) level detection}

Intracellular GSH level was measured with the standard GSH detection assay kit (Nanjing Jiancheng Bioengineering Institute, China) according to the manufacturer's instruction. The HCM cells $\left(1 \times 10^{5} /\right.$ well $)$ were seeded into 24-well plates and cultured overnight. Then, treated cells were collected and broken, supernatants were well mixed with precipitant, buffer and developer. After standing for $5 \mathrm{~min}$, the absorbance at $405 \mathrm{~nm}$ was measured using a microplate reader. The levels of GSH in left ventricular myocardium were measured following the described protocol above.

\section{Lactate dehydrogenase (LDH) activity assay}

The activity of LDH was assessed with the standard LDH activity assay kit (Nanjing Jiancheng). Cell culture supernatants or serum $(20 \mu \mathrm{L})$ were collected and added to master reaction mix according to the manufacturer's instruction. Five minutes after reaction, the absorbance at $450 \mathrm{~nm}$ was analyzed with the reader.

\section{Glutathione peroxidase 4 (GPx4) activity detection}

The lysates of myocardium were collected for GPx4 activity measurement by using an enzyme-linked immunosorbent assay (ELISA) kit (Shanghai Jianglai Biotechnology Co., Ltd., China). In short, equal amount $(50 \mu \mathrm{L})$ of standards and samples were added into corresponding wells and incubated for one hour at $37^{\circ} \mathrm{C}$ followed by addition of $50 \mu \mathrm{L}$ reaction substrate for $15 \mathrm{~min}$ incubation in dark at $37{ }^{\circ} \mathrm{C}$. After adding $50 \mu \mathrm{L}$ of termination solution into each well, the absorbance was analyzed at the wavelength of $450 \mathrm{~nm}$ with the microplate reader immediately.

\section{Lipid hydroperoxide (LPO) assay}

LPO level in cells or lysates of myocardium was determined with the standard kit (Nanjing Jiancheng). Briefly, lipid peroxide was incubated with chromogenic reagents under the condition of $45^{\circ} \mathrm{C}$ for $60 \mathrm{~min}$ followed by centrifugation for $10 \mathrm{~min}$. The absorbance of supernatants at $586 \mathrm{~nm}$ were measured with the microplate reader.

\section{RNA extraction and qRT-PCR}

Trizol reagent (Invitrogen, Missouri, USA) was used to isolate total RNAs from cultured cells or left ventricular myocardium tissues according to the manufacturer's instructions. DNaseI was included into the lysis buffer to avoid the contamination of DNA. $1 \mu \mathrm{g}$ total RNA of each sample was used for reverse transcription and then amplified by PCR with standard kits (Invitrogen, Missouri, USA). Relative expression levels of mRNA were normalized to GAPDH as internal controls. The following primers were used: ELAVL1: 5'-CGCAGAGATTCA GGTTCTCC-3' (forward), 5'-CCAAACCCTTTGCAC TTGTT-3' (reverse); GPx4: 5'-GTGGAACTTCACCAA GTTTGGAC-3' (forward), 5'-GGGCAGGTCCTTCTC TATCAC-3' (reverse); Beclin-1: 5'-GAGAACCTCAGC CGAAGACT- $3^{\prime}$ (forward), 5'-CCTCTAGTGCCAGCT CCTTT-3' (reverse); p62: 5'-GTACCAGGACAGCGA 
GAGGAA-3' (forward), 5'-CCCATGTTGCACGCC AAAC-3' (reverse); LC3: 5'-GAAGTTCAGCCACCT GCCAC-3' (forward), 5'-TCTGAGGTGGAGGGTCAG TC-3' (reverse); FOXC1: 5'-TAGCTACATCGCGCT CATCA-3' (forward), 5'-ACCTTGACGAAGCACTCG TT-3' (reverse); GAPDH: 5'-CCAGGTGGTCTCCTC TGA-3' (forward), 5'-GCTGTAGCCAAATCGTTGT-3' (reverse); ELAVL1 (mouse): $5^{\prime}$ - ACTGCAGGGATGACA TTGGG-3' (forward), 5'-CCAAGCTGTGTCCTGCTA CT-3' (reverse); Beclin-1 (mouse): 5'-CAGTGTTCCTGT GGAGTGGA-3' (forward), 5'-TGCACACAGTCCAGA AAAGC-3' (reverse); GAPDH (mouse): 5' - AGCCCA AGATGCCCTTCAGT-3' (forward), 5'-CCGTGTTCC TACCCCCAATG-3' (reverse).

\section{Western blotting}

RIPA lysis buffer (ThermoFisher, MI, USA) was utilized to extract proteins from left ventricular myocardium tissues or cells according to standard protocol. Protein concentration of each sample was measured by using Pierce $^{\mathrm{TM}}$ BCA Protein Assay Kit (ThermoFisher, MI, USA). Equal amounts of protein were loaded into SDSpolyacrylamide gels and separated through electrophoresis. Later the proteins were transferred from the gels to PVDF membranes (Sigma-Aldrich, USA). The membranes were blocked with 3\% BSA for half an hour at room temperature and then incubated with primary antibodies overnight at $4{ }^{\circ} \mathrm{C}$. On the next day the membranes were washed with TBST 3 times before incubation with specific secondary antibodies for $1 \mathrm{~h}$ at room temperature. Signals were detected by using the standard ECL kit. Primary antibodies used in the study were: Anti-ELAVL1 (1:1000; Abcam, USA); Anti-GPx4 (1:5000; Abcam); Anti-ferritin heavy chain (FTH1, 1:1000; Abcam); AntiBeclin-1 (1:1000; Santa Cruz, USA); Anti-p62 (1:1000; Abcam); Anti-LC3 (1:3000; Abcam); Anti-FOXC1 (1:1000; Abcam); Anti- $\beta$-actin (1: 1000; Santa Cruz).

\section{Chromatin immunoprecipitation (ChIP) Assay}

ChIP was performed using the commercial ChIP kit (Cell signaling technology, USA) according to the manufacturer's protocol. For each chromatin immunoprecipitation, $5 \mu \mathrm{g}$ of anti-FOXC1 and $1 \mu \mathrm{L}$ of normal rabbit IgG were used. IgG antibody was included as a negative control. After immunoprecipitation, chromosomal DNA was purified. ELAVL1 promoter region was detected by using qRT-PCR.

\section{RNA Immunoprecipitation (RIP) assay}

Transfected cells were lysed in lysis buffer $(50 \mathrm{mM}$ Tris-HCl, $150 \mathrm{mM} \mathrm{NaCl}, 2 \mathrm{mM}$ EDTA, 1\% NP-40, 0.5\% sodium deoxycholate) containing RNase inhibitors and protease inhibitors (Thermo Scientific, Waltham, MA,
USA). The extracted protein was incubated with relevant antibodies (anti-ELAVL1 and IgG as control) (Abcam, USA) overnight at $4{ }^{\circ} \mathrm{C}$ and then pulled down with protein G Sepharose 4 Fast Flow suspension (GE Amersham, Little Chalfont, UK). The beads were digested with proteinase K (Sangon, Shanghai, China) for $1 \mathrm{~h}$ followed by RNA purification with Trizol reagent (Invitrogen, Missouri, USA). qRT-PCR was performed to examine the RNA yield. The primers were listed in the qRT-PCR section.

\section{Biotin pull-down assay}

The biotinylated transcripts ( $3^{\prime}$ UTR of Beclin-1 mRNA, $5^{\prime} \mathrm{UTR}$, or the coding region) were prepared using the MEGshortscript $^{\mathrm{TM}}$ T7 kit based on the manufacturer's protocol and then incubated with cell lysates from transfected myocardial cells at $4{ }^{\circ} \mathrm{C}$ for $1 \mathrm{~h}$ followed by mixture with streptavidin-coupled dynabeads (Invitrogen, Shanghai, China) at $4{ }^{\circ} \mathrm{C}$ for another $3 \mathrm{~h}$. The beads were then washed with TENT (10 mM Tris- $\mathrm{HCl}[\mathrm{pH} 8.0], 1 \mathrm{mM}$ EDTA [pH 8.0], $250 \mathrm{mM} \mathrm{NaCl}, 0.5 \%$ Triton X-100) buffer 3 times and eluted with $1 \times$ laemmli SDS sample buffer. The elution was heated for $5 \mathrm{~min}$ at $95{ }^{\circ} \mathrm{C}$ and proceeded to western blotting.

\section{RNA stability}

For RNA stability assay, cells with treated with actinomycin D $(5 \mu \mathrm{g} / \mathrm{mL})$ for $0,2,4 \mathrm{~h}$ followed by RNA extraction using TRIzol reagent. Then, qRT-PCR was performed to assess mRNA remaining.

\section{Immunostaining}

Cells were fixed in $4 \%$ paraformaldehyde (PFA) at room temperature for $10-15 \mathrm{~min}$ and permeabilized with $0.1 \%$ Triton X-100 in PBS for half an hour at room temperature. Then cells were blocked with $1 \%$ BSA in PBS for $1 \mathrm{~h}$ at room temperature followed by incubation with primary antibody anti-LC3 (1: 500; Thermo Fisher Scientific, USA) at $4{ }^{\circ} \mathrm{C}$ overnight. Cells were then washed with PBS and incubated with secondary antibody conjugated with TRITC conjugated secondary antibody for $2 \mathrm{~h}$ at room temperature. DAPI was used to stain nucleus. Images were acquired with standard microscope.

\section{Dual luciferase reporter assay}

Fragments of the cDNAs containing the promoter region of ELAVL1 were amplified by PCR and were cloned into the Sall and bamHl restriction sites (Promega, USA) of the luciferase report gene of pmirGLO. Myocardial cells were cultured in 24-well culture plates for $12 \mathrm{~h}$ and then recombinant plasmids were co-transfected together with FOXC1 or empty vector into cells. The co-transfected cells were lysed using the Reporter Lysis Buffer. The 
luciferase activity of each sample was measured using the Dual-Luciferase Reporter Assay System (Promega, WI, USA).

\section{Infarct size measurement}

The infarct size was determined by 2,3,5-triphenyltetrazolium chloride (TTC) staining. Firstly, $1 \mathrm{~mL} \mathrm{1 \%} \mathrm{Evans}$ blue dye in $0.9 \% \mathrm{NaCl}$ was injected via the aorta to visualize the area at risk (AAR), and the hearts were immediately stored at $-20{ }^{\circ} \mathrm{C}$ for $1 \mathrm{~h}$ followed by coronally sectioning at $2 \mathrm{~mm}$. The sections were then stained with $1 \%$ TTC for $10 \mathrm{~min}$ at $37^{\circ} \mathrm{C}$. Images were analyzed with Image and live area, infarct area, AAR were measured by two blinded, independent operators. Lastly, infarct size was prestented as the percentage of AAR.

\section{Statistical analysis}

All experiments were performed with at least three biological replicates and the data were presented as Mean \pm SD. All statistical analyses were analyzed in GraphPad Prism 7. Statistical significance was determined by unpaired Student $t$ test or one-way ANOVA followed by Tukey's post test as indicated in figure legends. $P<0.05$ was considered as statistically significant.

\section{Results}

\section{Myocardial I/R induces ferroptosis and increases ELAVL1} expression level

To study the role of ferroptosis and ELAVL1 in myocardial I/R injury, we established the mouse myocardial I/R model. As shown in Fig. 1a with the TTC staining, myocardial I/R surgery caused a remarkable infarct area in the heart compared to the sham surgery (Fig. 1a, b). Then, we measured several parameters of ferroptosis, including LDH and GPx4 activity, FTH1, iron, and GSH levels. The results showed that I/R surgery significantly up-regulated LDH activity and cellular iron levels but greatly decreased GPx4 activity, FTH1 and GSH levels (Fig. 1c-h), indicating that ferroptosis occurs during myocardial I/R. Further, we found that ELAVL1 was remarkably elevated following I/R injury (Fig. 1d). These data show that ferroptosis is involved in myocardial $I / R$ injury and ELAVL1 may play a pivotal role during this process.

\section{Knockdown of ELAVL1 suppresses ferroptosis and H/R injury}

To test the role of ELAVL1 in myocardial I/R injury, we decreased ELAVL1 level by siRNA and measured its ensuring effects. We mimicked the myocardial I/R injury in vitro by exposing the cultured myocardial cells to hypoxia followed by reoxygenation. Consistently, H/R exposure increased ELAVL1 mRNA and protein levels but decreased GPx4 levels in myocardial cells (Fig. 2a-c). Transfection of cells with si-ELAVL1 suppressed the H/R induced upregulation of ELAVL1, as expected (Fig. 2ac). Also, knockdown of ELAVL1 recovered the mRNA and protein levels of GPx4 (Fig. 2a-c). With CCK-8 assay, we showed that $H / R$ substantially decreased the viability of myocardial cells while knockdown of ELAVL1 partially restored (Fig. 2d). In addition, $\mathrm{H} / \mathrm{R}$ exposure enhanced the levels of intracellular ROS, iron, LPO levels, and LDH activity but decreased cellular GSH level (Fig. 2e-j). However, knockdown of ELAVL1 restored those levels caused by H/R (Fig. $2 \mathrm{e}-\mathrm{j}$ ). Taken together, these results make clear that knockdown of ELAVL1 suppresses H/R induced ferroptosis and thus ameliorates cell injury.

\section{ELAVL1 regulates $H / R$ induced autophagy}

Ferroptosis is closely related to autophagy. To study how ELAVL1 regulated ferroptosis, we examined the level of autophagy during $H / R$ injury. $H / R$ exposure drastically increased the mRNA and protein levels of Beclin-1 and LC3 but diminished p62 levels (Fig. 3a-c). Interestingly, Knockdown of ELAVL1 in myocardial cells reverse those changes induced by $\mathrm{H} / \mathrm{R}$ (Fig. $3 \mathrm{a}-\mathrm{c}$ ). Moreover, with immunostaining of LC3 signal, we found H/R exposure greatly raised the intracellular level of LC3 while knockdown of ELAVL1 suppressed that rise (Fig. 3d, e). Therefore, we conclude that knockdown of ELAVL1 inhibits $H / R$ induced autophagy, which might be the mechanism of its anti-ferroptosis effect.

\section{Inhibition of autophagy suppresses H/R induced ferroptosis and cell injury}

To directly test the relationship between autophagy and ferroptosis during myocardial I/R injury, we used the autophagy inhibitor, 3-methyladenine (3-MA), and assessed its effects on $H / R$ induced ferroptosis and cell damage. As expected, 3-MA treatment significantly suppressed the increases of Beclin-1 and LC3 in protein caused by $H / R$, but recovered the levels of p62 (Fig. 4a, b), suggesting that $H / R$ induced autophagy was restrained by 3-MA. The mRNA and protein levels of GPx4 in cells exposed to $H / R$ were partially restored to the baseline by 3-MA (Fig. 4c-e). Further, 3-MA treatment recovered the reduced cell viability of $H / R$ exposed cells (Fig. 4f), while $H / R$ induced increase of cellular ROS level was suppressed by 3-MA treatment (Fig. 4g, h). Consistently, the changes of cellular iron, LPO, GSH levels, and LDH activity caused by $\mathrm{H} / \mathrm{R}$ were all restored to baseline when cells were treated with 3-MA (Fig. 4i-1). Al-together, these data provide an evidence that inhibition of autophagy could suppress H/R-induced ferroptosis and myocardial cell injury. 


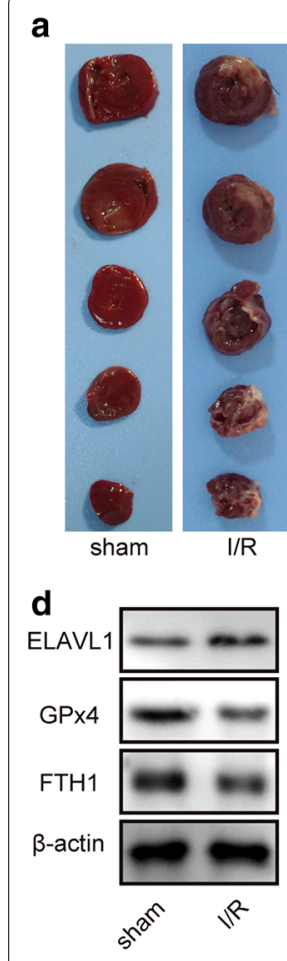

f

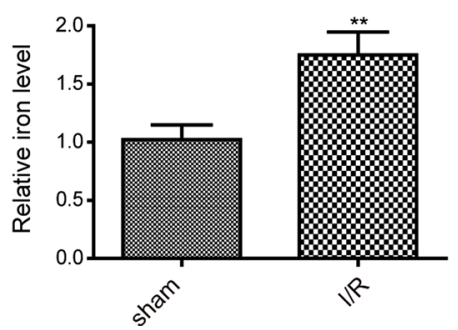

b

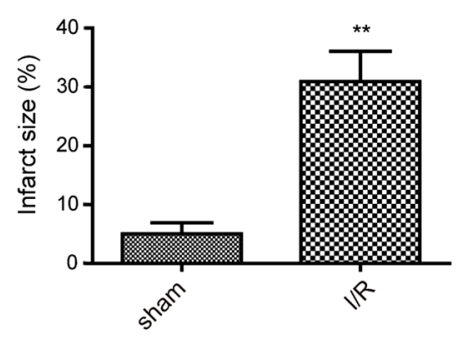

c

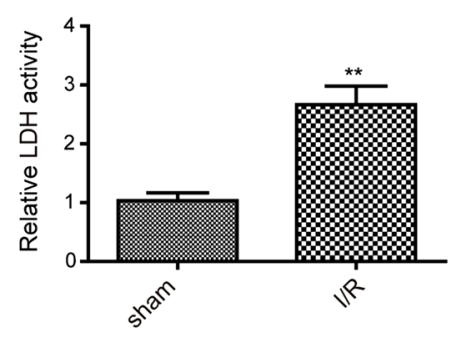

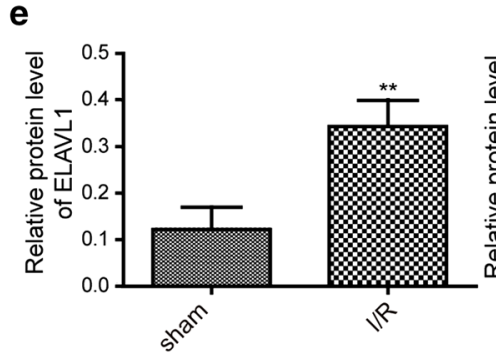

g
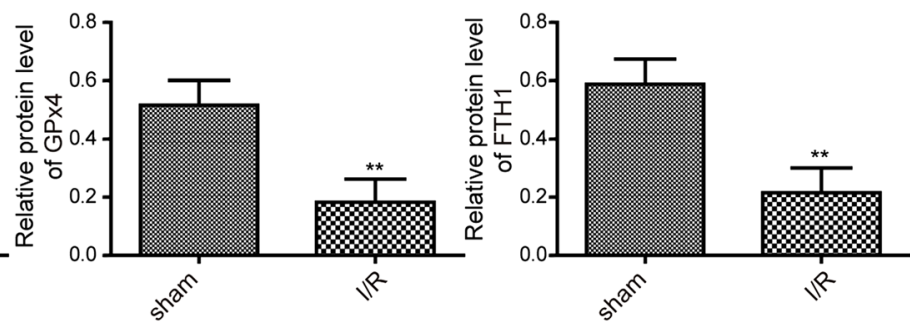

h

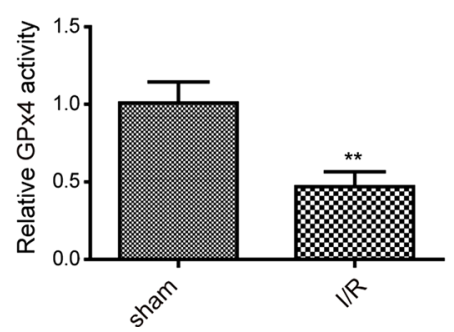

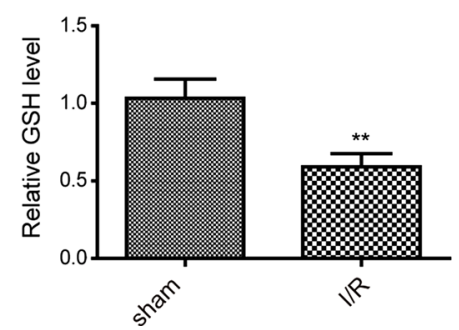

Fig. 1 Myocardial I/R induces ferroptosis and increases ELAVL1 expression level. Mice were subjected to myocardial I/R or sham surgery. a Representative TTC staining images of myocardial sections from sham-surgery mice and I/R surgery mice. $\mathbf{b}$ Quantifications of infarct size in each group. c Relative LDH activity in sham-surgery group and I/R group. d, e Relative protein levels of ELAVL1, GPX4, and FTH1 in sham-surgery group and I/R group. $\mathbf{f}$ Relative cellular iron levels in sham-surgery group and I/R group. $\mathbf{g}$ Relative GPx4 activity in sham-surgery group and I/R group. $\mathbf{h}$ Relative cellular GSH level in sham-surgery group and I/R group. The results were presented as the mean $\pm S D . n=5$; ${ }^{*} P<0.01$

\section{Knockdown of ELAVL1 suppresses I/R induced ferroptosis} and injury via inhibiting autophagy

Then we investigated the mechanisms underlying ELAVL1 anti-ferroptosis during $I / R$, and we focused on the autophagy as our aforementioned results showed that ELAVL1 restrained autophagy as well. As expected, knockdown of ELAVL1 through siRNA reversed the changes of p62, GPx4, Beclin-1, and LC3 induced by I/R surgery via up-regulating p62 and GPx4 and down-regulating Beclin-1 and LC3 (Fig. 5a-f). However, co-overexpression of Beclin-1, which promoted autophagy, suppressed the effects of si-ELAVL1
(Fig. 5a-f). Similarly, the increases of cellular iron level, LDH activity and LPO level induced by I/R surgery were inhibited by si-ELAVL1 (Fig. 5g-i). Cooverexpression of Beclin-1 in si-ELAVL1-treated mice increased those levels again upon I/R surgery (Fig. 5gi). Finally, knockdown of ELAVL1 via si-ELAVL1 significantly reduced the infarct size of myocardia (Fig. 5j, k). Nevertheless, overexpression of Beclin-1 together with si-ELAVL1 brought up the infarct size again (Fig. 5j, k). Taken together, these results prove that knockdown of ELAVL1 suppresses ferroptosis and ameliorates I/R injury through inhibiting autophagy. 

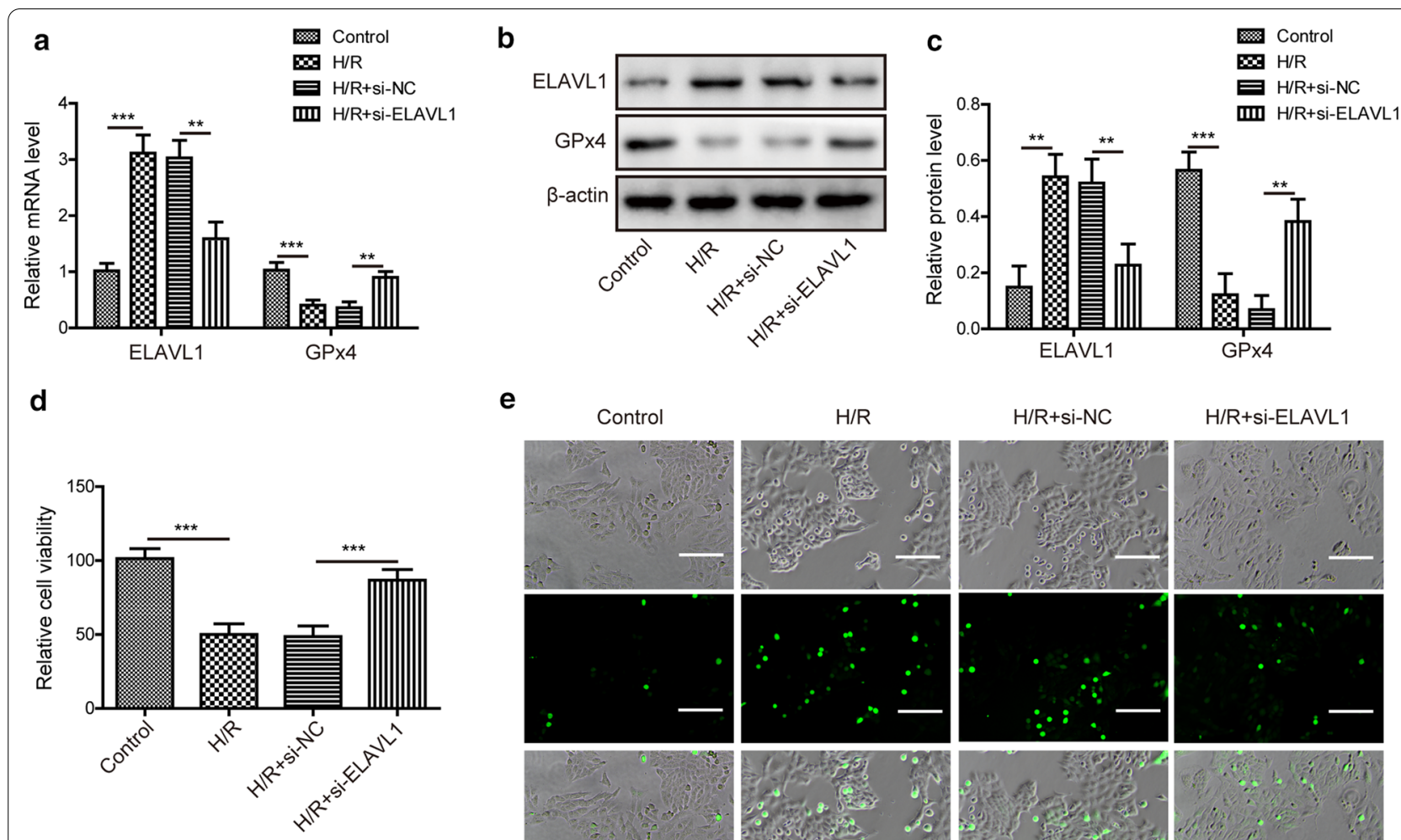

e
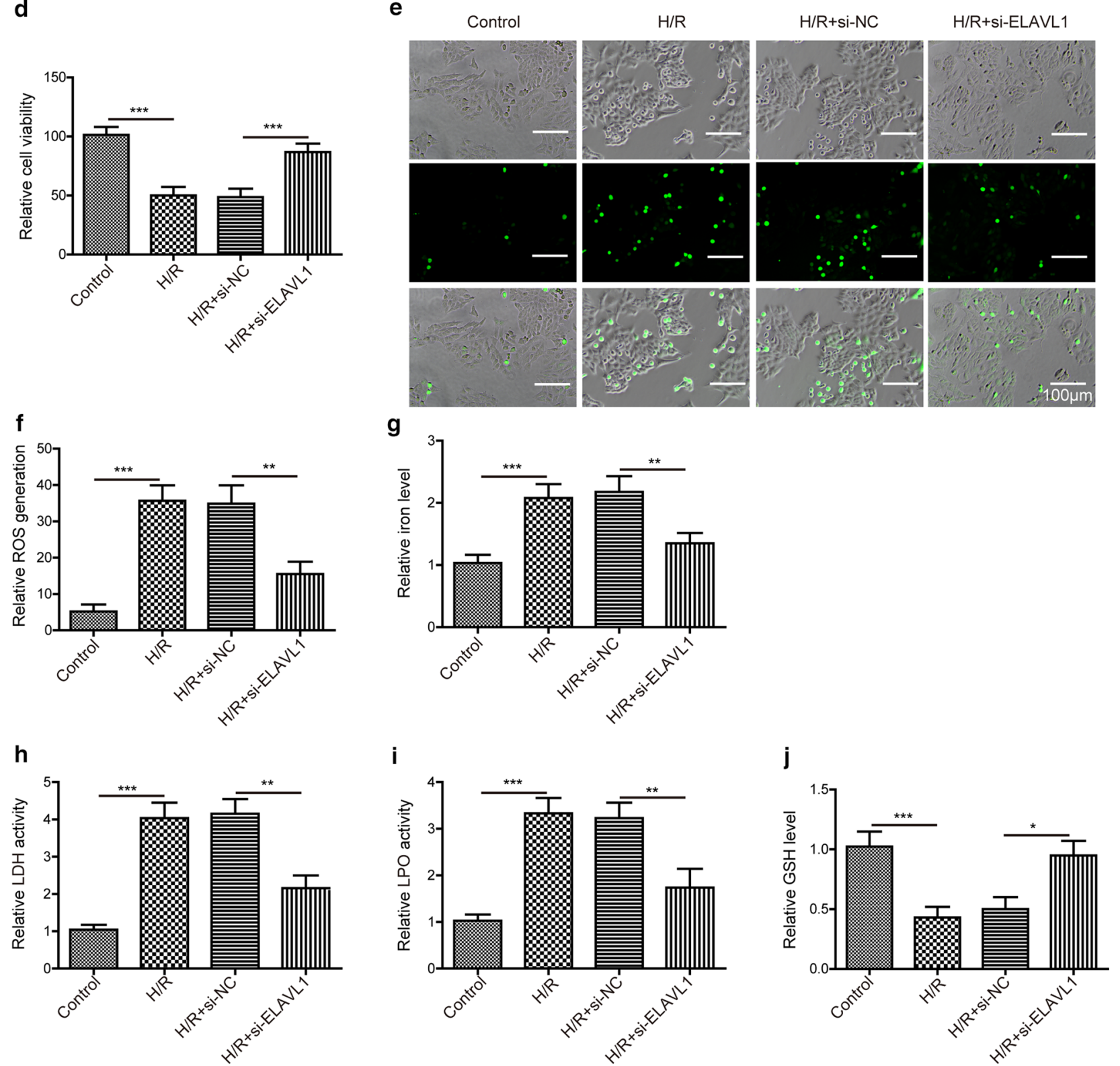

Fig. 2 Knockdown of ELAVL1 suppresses ferroptosis and H/R injury. Myocardial cells were transfected with si-ELAVL1 or si-NC and then subjected to H/R. a Relative mRNA levels of ELAVL1 and GPX4 in transfected myocardial cells following H/R exposure. b, c Relative protein levels of ELAVL1 and GPx4 in transfected myocardial cells following H/R exposure. $\mathbf{d}$ CCK8 assay to assess viability of transfected cells upon H/R exposure. e, $\mathbf{f}$ Relative cellular ROS levels were measured in each group of cells. Relative cellular iron level (g), LDH activity (h), LPO level (i) and GSH level (j) in each group were detected respectively. The results were presented as the mean \pm SD. $n=3 ;{ }^{*} P<0.05,{ }^{* *} P<0.01,{ }^{* * *} P<0.001$ 

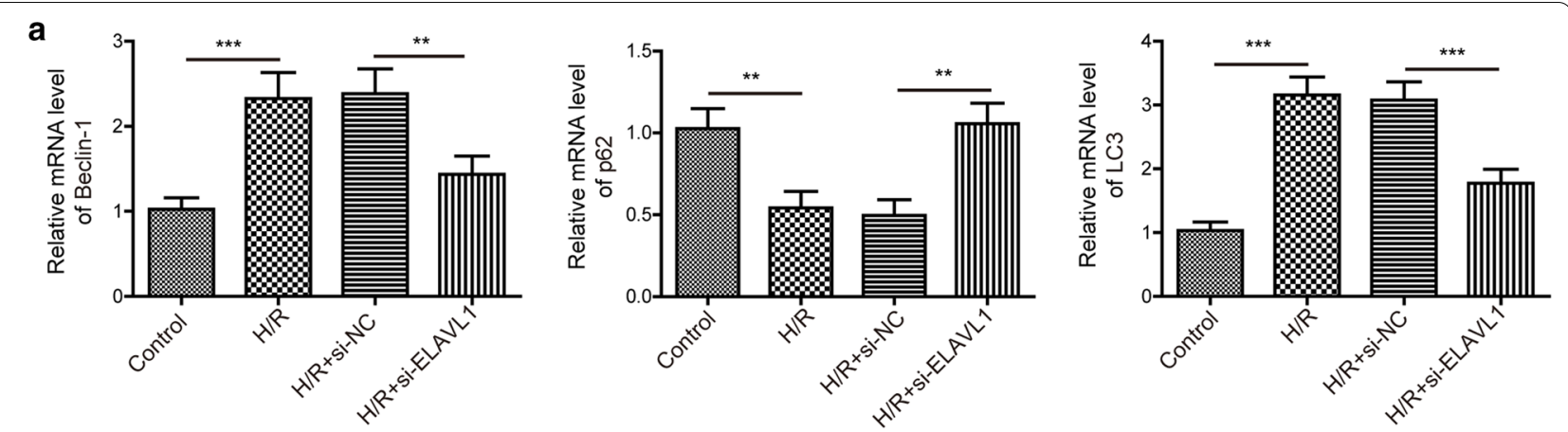

b

C
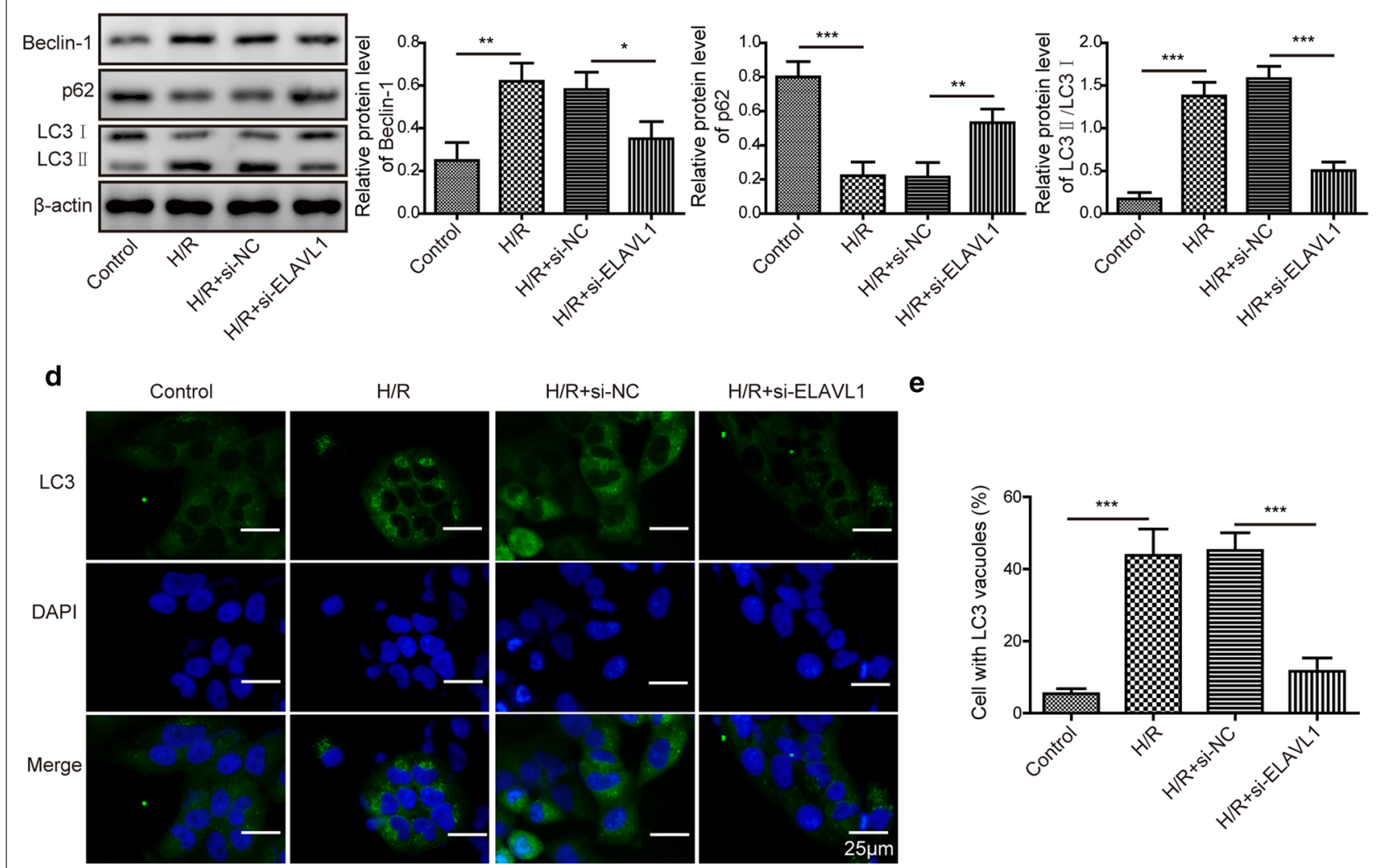

Fig. 3 ELAVL1 regulates H/R induced autophagy. Myocardial cells were transfected with si-ELAVL1 or si-NC and then subjected to H/R. a Relative mRNA levels of Beclin-1, p62 and LC3 were detected by qRT-PCR. $\mathbf{b}$, c Western blotting was allowed to assess relative protein levels of Beclin-1, p62 and LC3 in transfected cells following H/R. d, e Immunostaining analysis of cellular LC3 levels in transfected cells following H/R. The results were presented as the mean \pm SD. $n=3 ;{ }^{*} P<0.05,{ }^{* *} P<0.01,{ }^{* * *} P<0.001$

\section{ELAVL1 directly binds and stabilizes Beclin-1 mRNA}

Previous study suggests that ELAVL1 directly binds to Beclin-1 mRNA in hepatic stellate cells to regulate autophagy (Zhang 2018). We wondered whether similar interaction existed in myocardial cells. Using RIP assay, we found that ELAVL1 antibody pulled down significantly more Beclin-1 mRNA but not GAPDH mRNA compared to control IgG antibody (Fig. 6a), suggesting that ELAVL1 directly interacts with Beclin-1 mRNA in myocardial cells. We further characterized the binding region using biotinylated transcripts. As shown in Fig. 6b, we found that ELAVL1 bound with $3^{\prime}$ UTR of Beclin-1 mRNA, but not $5^{\prime}$ UTR or the coding region. We also examined how the binding affected the Beclin-1 mRNA stability. In control si-NC-transfected myocardial cells, application of the transcription 

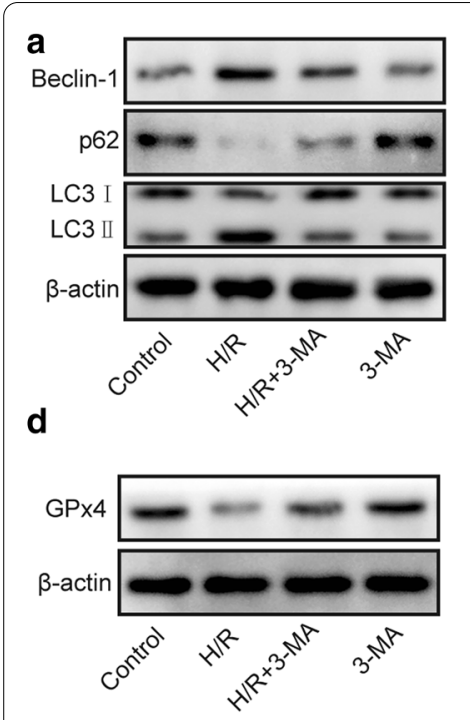

b

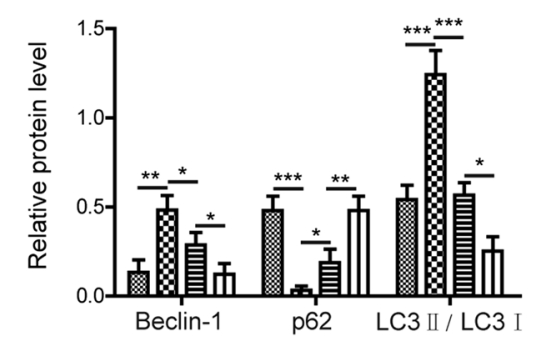

e

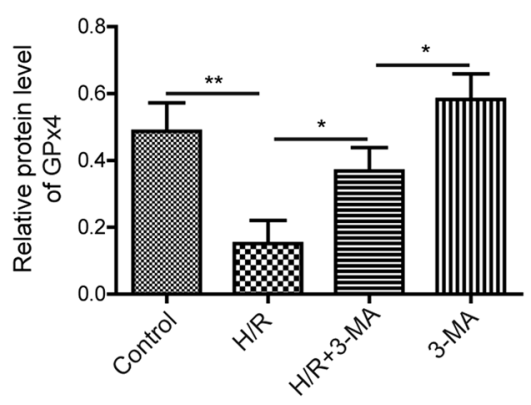

g Control

$H / R$

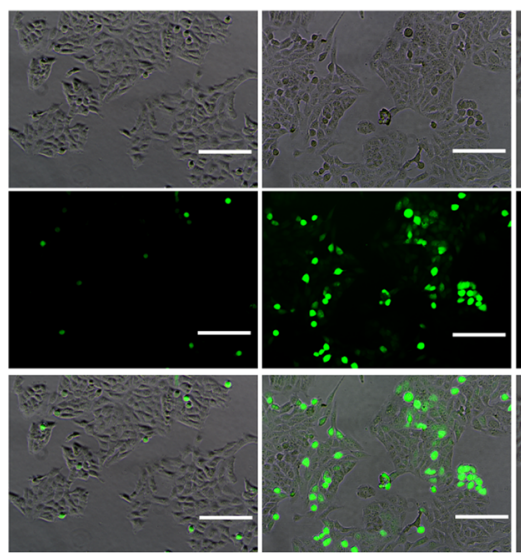

j

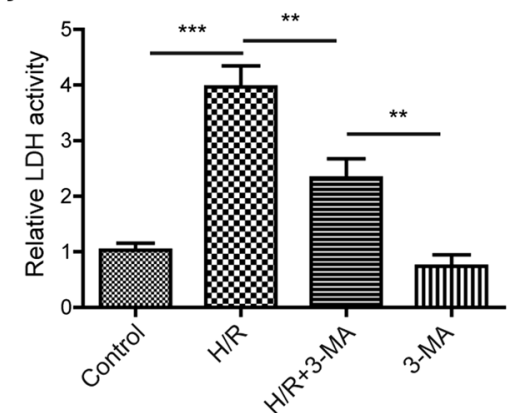

$H / R+3-M A$

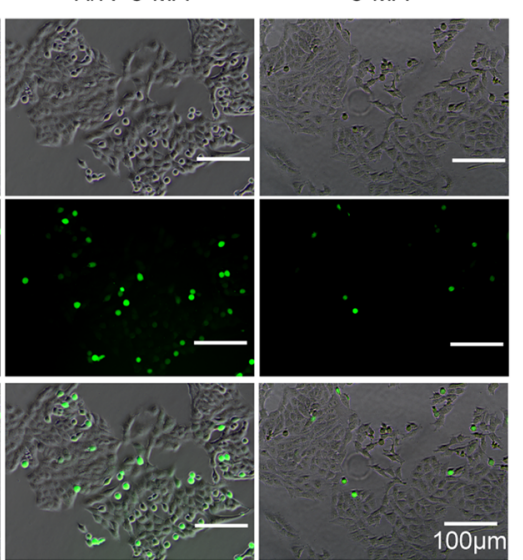

k

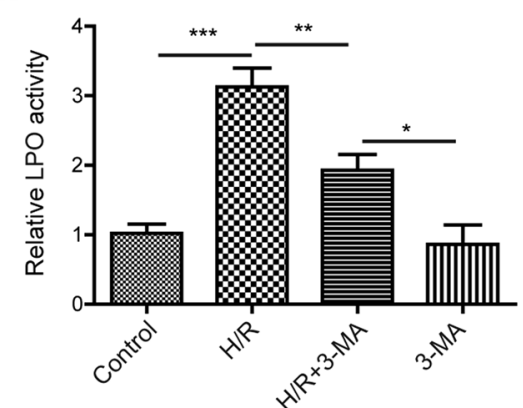

c

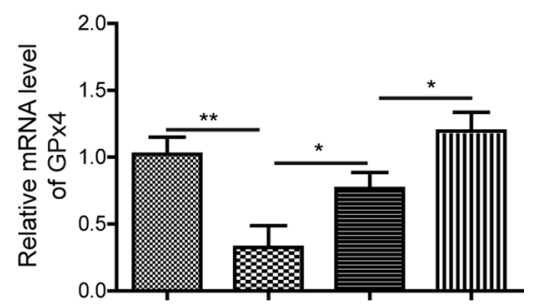

f

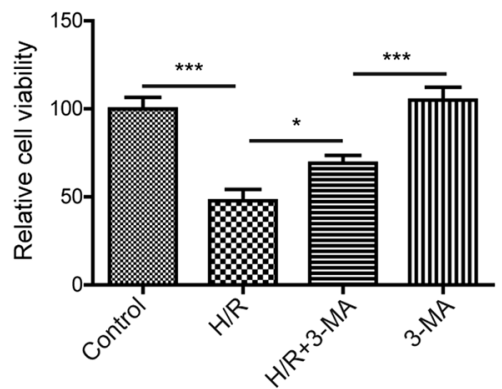

h

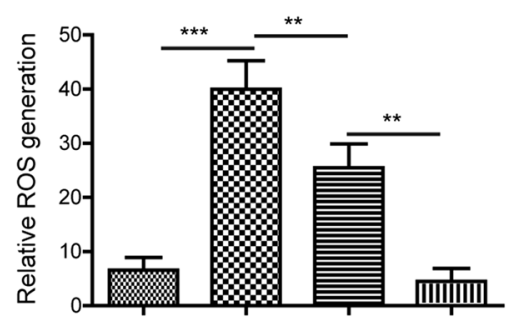

i
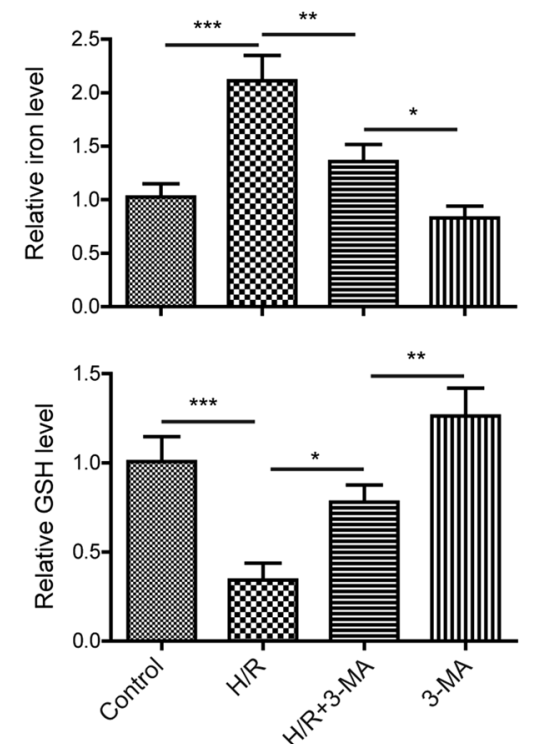

Fig. 4 Inhibition of autophagy suppresses H/R induced ferroptosis and cell injury. Myocardial cells were pretreated with 3-MA (5 mM) for $24 \mathrm{~h}$ before being subjected to H/R. a, b Relative protein levels of Beclin-1, p62 and LC3 were determined by western blotting. c-e Relative mRNA and protein levels of GPX4 in cells were assessed respectively. $\mathbf{g}$ CCK8 assay was performed to assess cell viability. (G\&H) Relative cellular ROS levels in 3-MA treated cells were measured after H/R. Relative cellular iron level (i), LDH activity (j), LPO level (k) and GSH level (I) in each group of cells were detected by corresponding assay kits after $\mathrm{H} / \mathrm{R}$. The results were presented as the mean $\pm \mathrm{SD} . \mathrm{n}=3 ;{ }^{*} P<0.05$, ${ }^{* *} P<0.01,{ }^{* * *} P<0.001$ 
(See figure on next page.)

Fig. 5 Knockdown of ELAVL1 suppresses I/R induced ferroptosis and injury via inhibiting autophagy. For inhibition of ELAVL1 or overexpression of Beclin-1, mice were received injection of lenti-si-ELAVL1 or Beclin-1. a Relative mRNA levels of ELAVL1 and Beclin-1 were measured by qRT-PCR. $\mathbf{b}-\mathbf{f}$ Relative protein levels of p62, GPx4, Beclin-1, and LC3 were determined by western blotting. Relative iron level $\mathbf{g}$, LDH activity $\mathbf{h}$ and LPO level $\mathbf{i}$ were assessed by corresponding assay kits. $\mathbf{j}$ Representative TTC staining images of myocardial sections from each group of mice. $\mathbf{k}$ Quantifications of infarct size in each group of mice. The results were presented as the mean $\pm S D . n=5 ;{ }^{*} P<0.05,{ }^{* *} P<0.01,{ }^{* * *} P<0.001$

inhibitor, actinomycin $\mathrm{D}$, gradually diminished the Beclin-1 mRNA levels with time (Fig. 6c). However, in si-ELAVL1 transfected cell, the reduction in Beclin-1 mRNA induced by actinomycin D was consistently and significantly more than that in control cells (Fig. 6c). These findings indicate that ELVAL1 directly binds with and stabilize Beclin-1 mRNA.

\section{FOXC1 activates ELAVL1 during $\mathrm{H} / \mathrm{R}$}

FOXC1 is a transcription factor that has been implicated in myocardial I/R injury (Zhang 2019). To test the mechanisms underlying ELAVL1 elevation following I/R surgery, we tested whether FOXC1 regulated ELAVL1 transcription. Exposure myocardial cells to $\mathrm{H} / \mathrm{R}$ increased FOXC1 mRNA level, as well as protein level (Fig. $7 \mathrm{a}-\mathrm{c}$ ). Transfection of cells with si-FOXC1 substantially diminished mRNA and protein levels of FOXC1 and suppressed the increase of FOXC1 expression induced by H/R exposure (Fig. 7d-f). Interestingly, we observed that knockdown of FOXC1 in control cells also decreased ELAVL1 level and that H/R induced upregulation of ELAVL1 level was reversed by FOXC1 knockdown as well (Fig. 7d-f), suggesting that FOXC1 regulates ELAVL1 expression. To further investigate that, we performed ChIP experiment. Immunoprecipitation with specific FOXC1 antibody successfully pulled down ELAVL1 promoter but not $\alpha$-Satellite, suggesting that FOXC1 directly binds ELAVL1 promoter (Fig. 7 g, h). Moreover, following H/R exposure, FOXC1 antibody pulled down more ELAVL1, indicating a stronger binding (Fig. $7 \mathrm{~g}, \mathrm{~h}$ ). We next examined which binding site mediated the regulation as we found two potential binding sites (E1 and E2) (Fig. 7i-k). Vectors containing the E1 binding site did not show any difference in luciferase activity compared to empty vector control while vectors containing the E2 binding site showed enhanced luciferase activity (Fig. 7j), suggesting that E2 is the functional binding site. Again, we observed a higher increase of luciferase activity following $H / R$ exposure (Fig. $7 k$ ), confirming that $H / R$ exposure enhances the binding. These results demonstrate that FOXC1 activates ELAVL1 transcription and H/R increases the transcription of ELAVL1 mediated by FOXC1.

\section{Discussion}

Cardiovascular disease like coronary heart disease is the top cause of death in the world, especially in the Western world (Shi et al. 2016). As the primary type of coronary heart disease, myocardial infarction affects millions of people worldwide (Aguero 2015; $\mathrm{Lu}$ et al. 2015). Currently the treatment of myocardial ischemia is limited and the damages are usually irreversible. Here, we studied the role of ferroptosis and ELAVL1 in myocardial I/R to provide insights into the molecular mechanisms. We showed that ferroptosis was induced and ELAVL1 was elevated following myocardial I/R surgery. Knockdown of ELAVL1 suppressed ferroptosis and myocardial I/R injury. Besides, suppression of ELAVL1 inhibited autophagy and inhibition of autophagy restrained ferroptosis and myocardial I/R injury. Molecularly, we identified that ELAVL1 bound with Beclin-1 mRNA and stabilized it. FOXC1 bound to ELAVL1 promoter region and activated ELAVL14 transcription during myocardial I/R. Activation of autophagy reversed the effects of ELAVL1 knockdown on ferroptosis and myocardial I/R injury. Our study sheds light on the molecular mechanisms of myocardial $\mathrm{I} / \mathrm{R}$ injury, providing avenues for the development of future therapeutic strategies.

Ferroptosis is a recently defined iron-dependent cell death (Mou 2019). Factors, such as suppressing system Xc- or GPx4, regulate glutathione peroxidase and decrease the antioxidant capacity, leading to accumulation of lipid ROS and eventually oxidative cell death ( $\mathrm{Li}$ 2020; Lei et al. 2019). Ferroptosis has been implicated in pathological conditions including neurodegenerative diseases (Alzheimer's and Parkinson's diseases), carcinogenesis, and kidney degeneration (Li 2020; Han 2020). Recently some studies reported ferroptosis in I/R injury (Li et al. 2020; Kobayashi 2018), but the exact function is not clear. Here, we provided strong evidence that myocardial I/R surgery induced substantial ferroptosis in the myocyte. We observed ferroptosis-specific changes including increases of cellular iron level and ROS signaling accompanied by decreased GPx4 and GSH levels following myocardial $I / R$ injury or $H / R$ exposure. Further, we showed that ferroptosis played an active and important role in the myocardial I/R injury. Inhibition of ferroptosis tremendously reduced the 


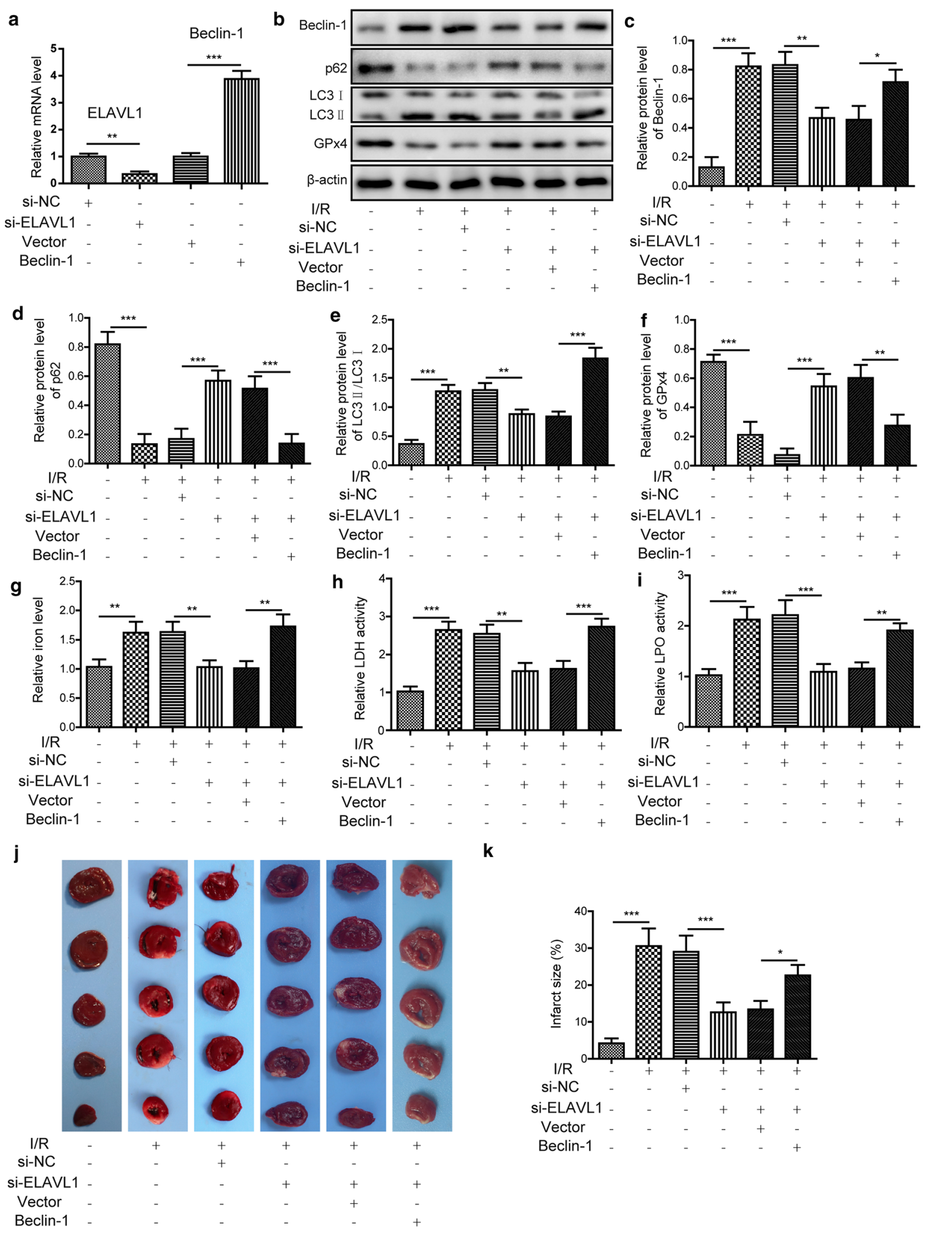



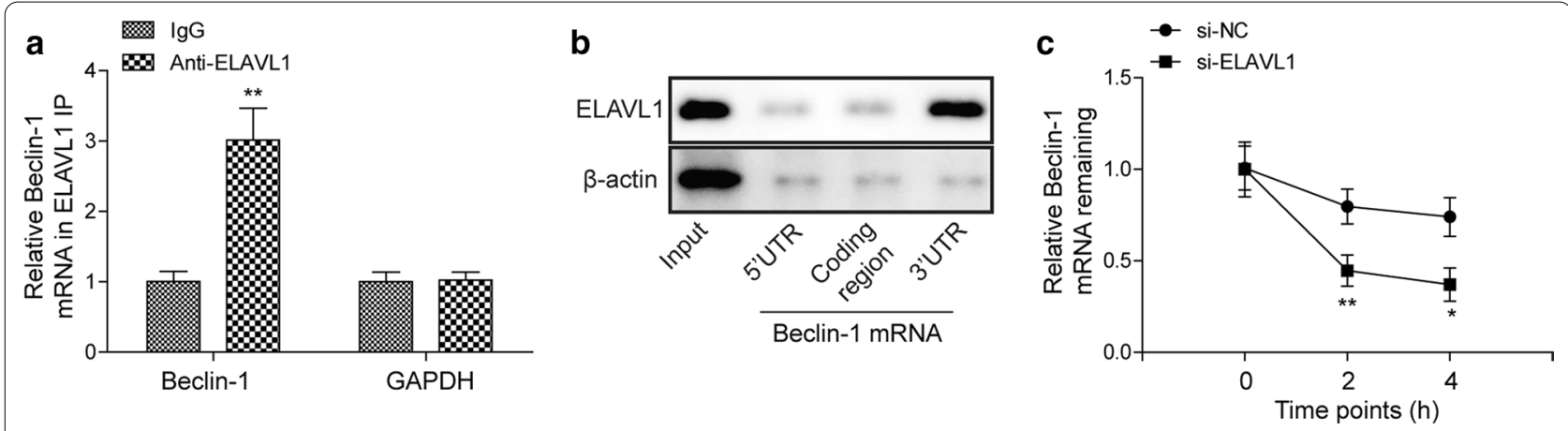

Fig. 6 ELAVL1 directly binds and stabilizes Beclin-1 mRNA. a Relative Beclin-1 mRNA and GAPDH mRNA levels following ELAVL1

immunoprecipitation. $\mathbf{b}$ Relative ELAVL1 protein level following biotin pull-down. c Relative Beclin-1 mRNA levels in transfected cells following actinomycin D treatment. The results were presented as the mean $\pm S D . n=3 ;{ }^{*} P<0.05,{ }^{* *} P<0.01$

injury. Therefore, ferroptosis could serve as a target for protection against myocardial I/R injury (Fang 2019).

ELAVL1 is a ubiquitous RBP and a key regulator of cytoplasmic mRNA fate (Chang and Hla 2014). Despite its primary location in nucleus, ELAVL1 translocates to cytoplasm to stabilize ARE-containing mRNAs and promote their expression upon cellular activation or stress conditions like oxidative stress and autophagy (Chang and Hla 2014). Numerous mRNAs have been shown to bind with ELAVL1, such as BCL2 (apoptosis regulator), HIF1A (hypoxia inducible factor 1 subunit alpha), TNF (tumor necrosis factor), NOS2 (nitric oxide synthase 2) (Chang 2014, 2013; Skliris 2015). Therefore, ELAVL1 is largely involved in many processes including apoptosis, autophagy, and oxidative stress (Srikantan and Gorospe 2012; Katsanou 2009; Zucal 2015). Here, during myocardial $I / R$ injury wherein excessive ROS and inflammatory cytokines were produced (Zhou et al. 2015), we saw a substantial increase of ELAVL1. Further, this up-regulation plays a positive role in autophagy and ferroptosis in that knockdown of ELAVL1 greatly suppressed those processes, thus ameliorating I/R injury. Similar promoting role of ELAVL1 in ferroptosis has been reported during liver fibrosis (Zhang 2018), suggesting a conserved role of ELAVL1 in cell or tissue damage. Moreover, Beclin-1 mRNA has been shown as a target of ELAVL1 to trigger autophagy and ferroptosis in liver fibrosis (Zhang 2018). Consistently, inhibition of autophagy could suppress ferroptosis in H/R induced HCM cells and Beclin-1 could reversed the effects of ELAVL1 silencing on myocardial $I / R$ injury in mice via regulating autophagy and ferroptosis. We also confirmed a direct interaction between ELAVL1 and Beclin-1 mRNA and showed that this binding promotes Beclin-1 mRNA stability, which could be the mechanism through which ELAVl1 accelerates autophagy process. In addition, it will be interesting to examine if there are other targets mediating ELAVL1's role in autophagy and ferroptosis (Kang and Tang 2017).

The increase of ELAVL1 induced by myocardial I/R is mediated by FOXC1-activated transcription. FOXC1 is a transcription factor that plays an important role in heart and cardiovascular development (Lambers 2016; Kume 2009). Dysregulation of FOXC1 has been reported in diseases including congenital heart defects, cancers, and myocardial ischemia (Zhang 2019; Elian et al. 2018). FOXC1 expression could be induced by hypoxia and subsequently it can activate the expression of toll-like receptors, contributing to the inflammatory responses and cell injury (Zhang 2019). Here, we identified ELAVL1 as a novel target of FOXC1. FOXC1 directly bound to ELAVL1 promoter region and promotes ELAVL1 transcription. Furthermore, this interaction was enhanced following $H / R$ exposure, resulting higher level of ELAVL1. Notably, previous studies have shown that ELAVL1 could target FOXC1 mRNA to promote its stability (Katsanou 2009). This implies that ELAVL1/FOXC1 might form a positive feedback loop during H/R. Our study, together with previous work, show that FOXC1 is a key player in the myocardial I/R injury.

\section{Conclusions}

In summary, we showed that autophagy-dependent ferroptosis contributes to the myocardial I/R injury and that this ferroptosis is caused by ELAVL1, leading to overproduction of lipid signaling. Additionally, FOXC1 activates ELAVL1 transcription in myocardial I/R injury. Targeting ferroptosis or FOXC1/ ELAVL1 could be beneficial for myocardial ischemia patients. 


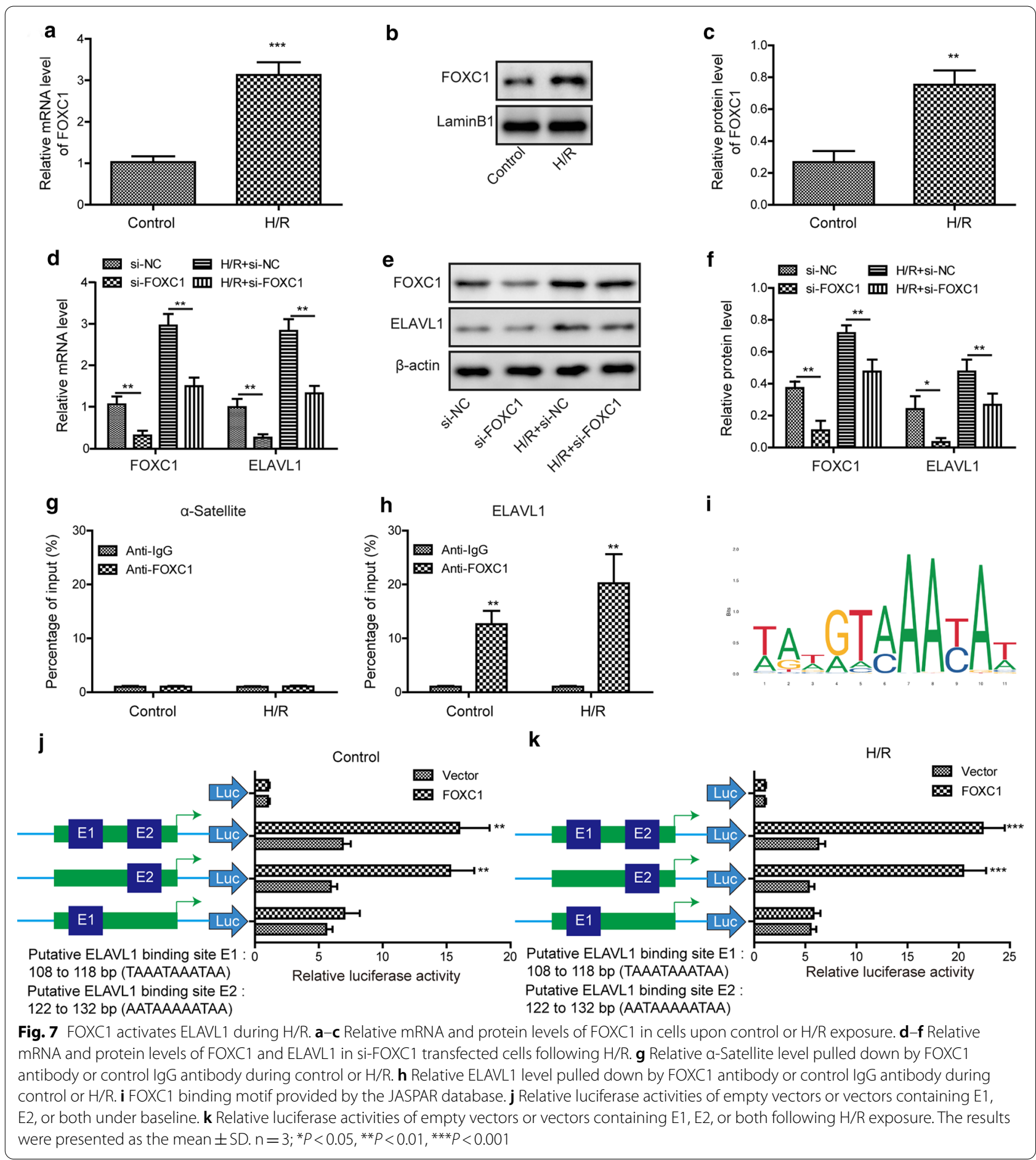

Acknowledgements

We would like to give our sincere gratitude to the reviewers for their constructive comments.

\section{Authors' contributions}

HYC: Conceptualization; ZZX: Funding acquisition; Writing —original draft; XL: Methodology; RNX: Data curation; Resources; PZ: Formal analysis; Investigation; Software; Visualization; SYZ: Project administration; Supervision;
Validation; Writing—review \& editing. All authors read and approved the final manuscript.

\section{Funding}

This work was supported by the President's fund of Southern Hospital of Southern Medical University (No.2017Z018). 


\section{Availability of data and materials}

All data generated or analyzed during this study are included in this article. The datasets used and/or analyzed during the current study are available from the corresponding author on reasonable request.

\section{Code availability}

Not Applicable.

\section{Ethical approval and consent to participate}

All animal experiments have been approved by the Committee on Animal Care and Use of Nanfang Hospital, Southern Medical University and were performed according to the guidance.

\section{Consent for publication}

Not Applicable. This article does not contain any studies with human participants performed by any of the authors.

\section{Competing interests}

The authors declare that there is no competing interests.

\section{Author details}

${ }^{1}$ Department of Cardiovascular Surgery, Nanfang Hospital, Southern Medical University, No.1838 North Guangzhou Avenue, Baiyun District, Guangzhou 510515, Guangdong, People's Republic of China. ${ }^{2}$ Department of Thoracic Surgery, Yuebei People's Hospital, Shantou University, Shaoguan 512026, Guangdong, People's Republic of China.

Received: 1 November 2020 Accepted: 11 January 2021

Published online: 10 February 2021

\section{References}

Aguero F, et al. New myocardial infarction definition affects incidence, mortality, hospitalization rates and prognosis. Eur J Prev Cardiol. 2015;22:1272-80.

Chang SH, Hla T. Post-transcriptional gene regulation by HuR and microRNAs in angiogenesis. Curr Opin Hematol. 2014;21:235-40.

Chang $\mathrm{SH}$, et al. Antagonistic function of the RNA-binding protein HuR and miR-200b in post-transcriptional regulation of vascular endothelial growth factor-A expression and angiogenesis. J Biol Chem. 2013;288:4908-21.

Chang SH, et al. ELAVL1 regulates alternative splicing of elF4E transporter to promote postnatal angiogenesis. Proc Natl Acad Sci U S A. 2014;111:18309-14.

de Bruin RG, Rabelink TJ, van Zonneveld AJ, van der Veer EP. Emerging roles for RNA-binding proteins as effectors and regulators of cardiovascular disease. Eur Heart J. 2017;38:1380-8.

Elian FA, Yan E, Walter MA. FOXC1, the new player in the cancer sandbox. Oncotarget. 2018;9:8165-78.

Fang $\mathrm{X}$, et al. Ferroptosis as a target for protection against cardiomyopathy. Proc Natl Acad Sci U S A. 2019;116:2672-80.

Frank A, et al. Myocardial ischemia reperfusion injury: from basic science to clinical bedside. Semin Cardiothorac Vasc Anesth. 2012;16:123-32.

Han C, et al. Ferroptosis and its potential role in human diseases. Front Pharmacol. 2020;11:239.

Hannenhalli S, Kaestner KH. The evolution of Fox genes and their role in development and disease. Nat Rev Genet. 2009;10:233-40.

Hausenloy DJ, Yellon DM. Myocardial ischemia-reperfusion injury: a neglected therapeutic target. J Clin Invest. 2013;123:92-100.

Kang R, Tang D. Autophagy and ferroptosis-what's the connection? Curr Pathobiol Rep. 2017;5:153-9.

Katsanou V, et al. The RNA-binding protein Elavl1/HuR is essential for placental branching morphogenesis and embryonic development. Mol Cell Biol. 2009;29:2762-76.
Kobayashi M, et al. Pathological roles of iron in cardiovascular disease. Curr Drug Targets. 2018;19:1068-76.

Krishnamurthy $\mathrm{P}$, et al. Myocardial knockdown of mRNA-stabilizing protein HuR attenuates post-MI inflammatory response and left ventricular dysfunction in IL-10-null mice. FASEB J. 2010;24:2484-94.

Kume T. The cooperative roles of Foxc1 and Foxc2 in cardiovascular development. Adv Exp Med Biol. 2009;665:63-77.

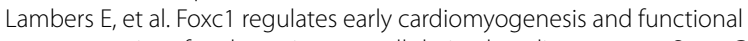
properties of embryonic stem cell derived cardiomyocytes. Stem Cells. 2016;34:1487-500.

Lei P, Bai T, Sun Y. Mechanisms of ferroptosis and relations with regulated cell death: a review. Front Physiol. 2019;10:139.

Levy NS, Chung S, Furneaux H, Levy AP. Hypoxic stabilization of vascular endothelial growth factor mRNA by the RNA-binding protein HuR. J Biol Chem. 1998;273:6417-23.

Li J, et al. Ferroptosis: past, present and future. Cell Death Dis. 2020;11:88.

Li W, Li W, Leng Y, Xiong Y, Xia Z. Ferroptosis is involved in diabetes myocardial ischemia/reperfusion injury through endoplasmic reticulum stress. DNA Cell Biol. 2020;39:210-25.

Lin YJ, et al. Tumor hypoxia regulates forkhead box C1 to promote lung cancer progression. Theranostics. 2017;7:1177-91.

Lu L, Liu M, Sun R, Zheng Y, Zhang P. Myocardial infarction: symptoms and treatments. Cell Biochem Biophys. 2015;72:865-7.

Mou Y, et al. Ferroptosis, a new form of cell death: opportunities and challenges in cancer. J Hematol Oncol. 2019;12:34.

Shi A, Tao Z, Wei P, Zhao J. Epidemiological aspects of heart diseases. Exp Ther Med. 2016;12:1645-50.

Simone LE, Keene JD. Mechanisms coordinating ELAV/Hu mRNA regulons. Curr Opin Genet Dev. 2013;23:35-43.

Skliris A, et al. Neuroprotection requires the functions of the RNA-binding protein HuR. Cell Death Differ. 2015;22:703-18.

Srikantan S, Gorospe M. HuR function in disease. Front Biosci (Landmark Ed). 2012;17:189-205.

Turer AT, Hill JA. Pathogenesis of myocardial ischemia-reperfusion injury and rationale for therapy. Am J Cardiol. 2010;106:360-8.

Wang J, et al. Multiple functions of the RNA-binding protein HuR in cancer progression, treatment responses and prognosis. Int J Mol Sci. 2013;14:10015-41.

Wu D, Zhang K, Hu P. The role of autophagy in acute myocardial infarction. Front Pharmacol. 2019;10:551.

Xie Y, et al. Ferroptosis: process and function. Cell Death Differ. 2016;23:369-79.

Zhang $Z$, et al. Activation of ferritinophagy is required for the RNA-binding protein ELAVL1/HuR to regulate ferroptosis in hepatic stellate cells. Autophagy. 2018;14:2083-103.

Zhang SP, et al. FOXC1 up-regulates the expression of toll-like receptors in myocardial ischaemia. J Cell Mol Med. 2019;23:7566-80.

Zhou T, Chuang CC, Zuo L. Molecular characterization of reactive oxygen species in myocardial ischemia-reperfusion injury. Biomed Res Int. 2015;2015:864946.

Zhou B, et al. Ferroptosis is a type of autophagy-dependent cell death. Semin Cancer Biol. 2019a. https://doi.org/10.1016/j.semcancer.2019.03.002.

Zhou Y, et al. The crosstalk between autophagy and ferroptosis: what can we learn to target drug resistance in cancer? Cancer Biol Med. 2019b;16:630-46.

Zucal C, et al. Targeting the multifaceted HuR protein, benefits and caveats. Curr Drug Targets. 2015;16:499-515.

\section{Publisher's Note}

Springer Nature remains neutral with regard to jurisdictional claims in published maps and institutional affiliations. 